\title{
Expression Toll-like receptors in the oral mucosa of patients with recurrent aphthous stomatitis
}

\author{
Diah Savitri Ernawati \\ Department of Oral Medicine \\ Faculty of Dentistry Airlangga University \\ Surabaya - Indonesia
}

\begin{abstract}
Toll-like receptors (TLRs) have recently emerged as key receptors of the innate immune system. They recognize specific pathogenassociated molecular patterns initiating a host defence response. The oral mucosa epithelium encounters potential pathogens like bacteria and viruses in inspired air, and the discovery of TLRs on epithelial cells suggest that the epithelium has a role in the mucosal immune system. The aim of this study was to discover Recurrent Apthous Stomatitis (RAS) using etiopathogenetic molecular approach by observing the TLRs. Immunohistochemistry using monoclonal antibodies anti-TLR-2, TLR-3, TLR-4, TLR-5, TLR-7 and TLR-9 were used in this study. These antibodies are specific toward TLR on the surface of epithelial cells membrane and macrophages in patients with major and minor RAS. TLR was expressed on the surface of epithelial cells membrane of oral mucosa and macrophages in both major and minor RAS patients. TLRs was not expressed specifically in non-RAS patients. The results above showed indication, that functional TLRs expression by epithelial cells in oral mucosa had remarkable implication on natural immune response and disease pathogenesis. The expression of TLR was found in the oral mucous membrane on epithelial cells surface and macrophages patients with RAS.
\end{abstract}

Key words: major and minor RAS, toll-like receptors

Correspondence: Diah Savitri Ernawati, c/o: Bagian Oral Medicine, Fakultas Kedokteran Gigi Universitas Airlangga. Jl. Mayjend. Prof. Dr. Moestopo no. 47 Surabaya 60132, Indonesia.

\section{INTRODUCTION}

The oral mucosal epithelium is an important interface with the environment, and represents a dynamic system for innate host defense. In addition to providing a barrier to the entry of pathogens, epithelial cells lining oral mucosal have been shown to response the presence of microorganisms by producing natural antimicrobial factors and mounting an inflammatory response. ${ }^{1,2}$

The innate immune system also plays a role in several human diseases. The primary function of Tolllike receptors (TLRs) is to recognized pathogens, TLRs can also specifically recognize pathogen-associated molecular patterns (PAMPs), tranduce signals into cells and initiate complex signal cascade leading to activation of the transcriptional factors such as nuclear factor kappa $\beta$ $(\mathrm{NF}-\kappa \beta)$ and interferon regulatory factor. ${ }^{3-5}$

Since 1996, 11 TLRs (TLRs1-11) have been found in both mammal and human dendrite, mononuclear and epithelial cells of intestine and bronchus, endothelial cells of blood vessels and epithelial cells as well as cells from various organ system. Toll-like receptors are transmembrane proteins, all of which have common extracellular leuinerich domain and conserved cytoplasmic domain. The cytoplasmic domain of TLR is homologous to the IL-1 and IL-18 receptors and contains the Toll/IL-1 receptor (TIR) homology domain common to these receptors. ${ }^{3-8}$
Recurrent aphthous stomatitis (RAS) are the most common oral disease characterized by repeated development of painful ulcers. The aetiology of RAS is still unknown. Many local and systemic factors such as bacterial or viral infection, genetic factor, hormonal, immune system disorders, hematinic deficiency, and systemic disease could be involved in pathogenesis of RAS. ${ }^{9-11}$ Previous studies have also suggested that this inflammatory disease is a result of abnormal immune response directed towards the oral mucosa. Activation of the immune system in aphthous lesions and peripheral blood was observed in RAS. ${ }^{3,13}$ Tissue-specific autoimmunity is one of the most probable mechanism of RAS development, with possible activation of Th1 lymphocyte profile of cytokine production. ${ }^{12-14}$

Stimulation of internal and external factors could induce multiplication process in proliferation cell phase. It eventually could be continuously express protein anomaly, because the superficial character is different from normal oral mucosa. ${ }^{15,16}$

Decrease ability of oral tolerance activities on inflammation of disease pathogenesis showed very impressively reduced of $\mathrm{T}$ helper, $\mathrm{Th}-2$, and $\mathrm{Th}-3$. Genetic and entvironment may contribute to lower tolerance caused cytotoxic effect by oral epithelial. ${ }^{17}$ The objective of this study was to disclose or discover RAS using etiopathogenetic molecular approach by observing the TLRs. Main objectives of this study was to prove 
the presence of TLRs on the surface of epithelial cells membrane and macrophages and to identify predominant TLRs types at those sites in patients with major and minor RAS.

\section{MATERIALS AND METHODS}

\section{Patients and samples}

Inform concernt approved by the Research Ethics Committee was obtained from all the participants. The study population consisted of 21 samples major RAS (10 women, 11 men) and 18 samples minor from RAS patients ( 7 men, 11 women, mean age 21 years; range 19-55 years) who did not have any systemic or inflammatory disease and 11 healthy individuals. RAS patients were defined as those having at least one aphthous lesion per month during the preceding years. Tissue samples were obtained from patients in an active stage of RAS (1-3 days old ulcers) and from controls were collected by using Fine Needle Aspiration Biopsy (FNAB) on ulcer marginal edge and scrapped of oral epithelial biopsy. Five $\mathrm{ml}$ of venous blood were drawn from the anterior arm, anti-coagulated with heparin and diluted 1:1 with Hank's liquid. Then the mixture was gently added to a centrifuge tube containing $5 \mathrm{ml}$ of lymphocyte-separating liquid and centrifuged at $700 \mathrm{r} / \mathrm{min}$ for 20 minutes. The cells in gray middle layer were collected and washed twice with PBS. The deposit contained about $25 \%$ monocytes and $75 \%$ lymphocytes. ${ }^{17}$

\section{Immunohistochemistry}

Scrapped specimen of oral epithelial biopsy in oral mucosa fixed on an object glass with $90 \%$ alcohol, and incubated in refrigerator or directly blocked with $1 \%$ bovine serum albumin (BSA 1\%) and incubated in $\mathrm{CO}_{2}$ at the temperature $37^{\circ} \mathrm{C}$ for $45 \mathrm{~min}$. After being washed with PBS, sample is reacted with monoclonal antibody TLRs anti TLR-2, TLR-3, TLR-4, TLR-5, TLR-7 and TLR-9, re-incubated in $\mathrm{CO}_{2}$ incubator at $37^{\circ} \mathrm{C}$ for one hour. After being washed with PBS, the sample was analyzed using immunofluorescence microscope by magnifying with $40 \times$.

\section{RESULTS}

A study has been conducted to 21 and 18 patients with major and minor RAS, respectively, and to 10 non-RAS patients as control in order to identify the presence of protein like receptors (TLRs) in epithelial cells and macrophages of patients with RAS. In this study, it was found that TLRs was expressed on the surface of epithelial cells membrane of oral mucosa and macrophages in both major and minor RAS patients. TLR was not expressed specifically in non-RAS patients. The patients with positive TLRs- 2 were $41.02 \%$, revealed among minor RAS patients, while in major RAS TLRs-2 was $43.58 \%$ expressed in (Figure 1).

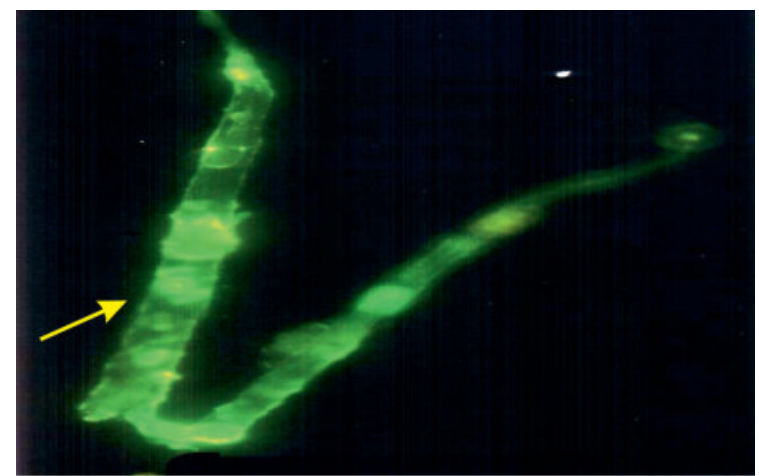

Figure 1. Immunohistochemical detection of TLRs in minor RAS patients with scrapped oral epithelial biopsy. TLRs-2 expression on cytoplasmic and surface of epithelial cell membrane.

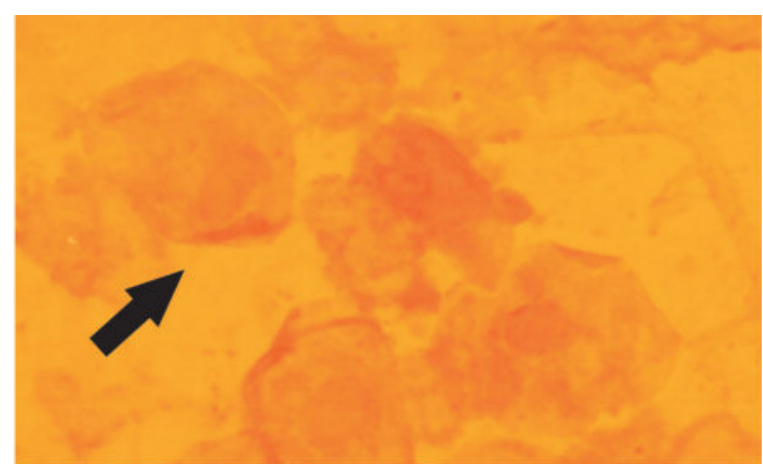

Figure 2. TLRs-3 expression on surface of epithelial cells membrane in the oral mucosal major RAS patients reacted with TLRs-3 monoclonal antibody. RAS major and minor visualized with DAB-chromogen

The samples which expressed TLRs-3 were mostly major RAS patients as much as $43.58 \%$ from 38 RAS patients, while those with minor RAS positive TLRs-3 was $17.94 \%$ (Figure 2).

RAS patients with positive TLRs- 4 was $48.71 \%$, found in major RAS patients, while in minor RAS TLRs-4 was $38.46 \%$ expressed (Figure 3).

Samples of RAS patients expressing TLRs-5 was $7.69 \%$ in major RAS patients, and $10.25 \%$ was expressed mostly in minor RAS patients. Immunofluorescent analysis showed that TLRs-5 was not well expressed in epithelial cell surface and macrophage The expression of TLRs-7 in RAS cases that had been analyzed immunohistochemically showed no specific expression either on the surface of cell membrane or cytoplasm. Only $20.51 \%$ showed positively against TLRs-7. RAS patients with positive TLRs-9 were $33.33 \%$. For major RAS cases, there were $17.94 \%$ with positive TLRs-9 and, similarly, there were also $17.94 \%$ positively minor RAS cases (Figure 4). 

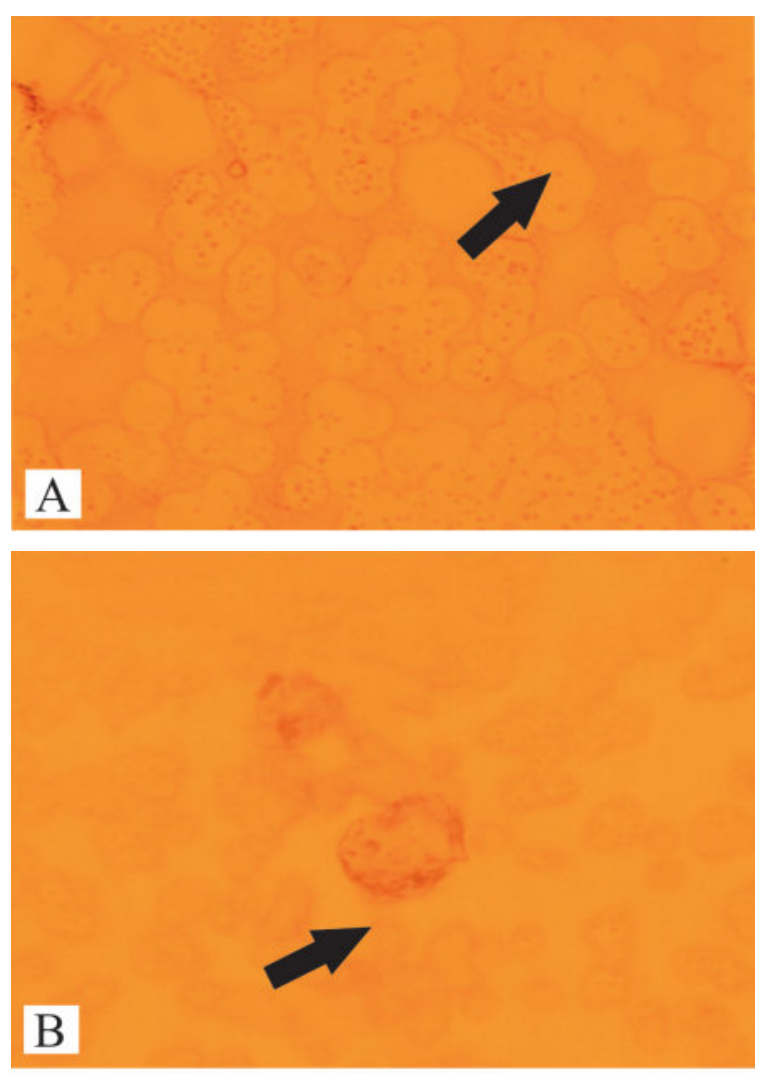

Figure 3. A) TLRs-4 expression in this minor RAS was not well ditributed in all cells, either on the cells membrane surface or in the cytoplasms. B) TLRs-4 expression in major RAS was found on epithelial surface and macrophages cells surface.

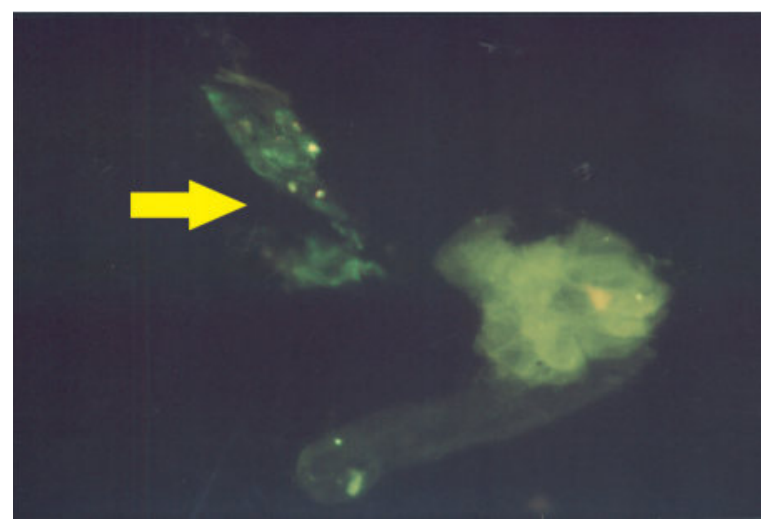

Figure 4. Scrapped oral epithelial biopsy in major RAS patients after reacted with TLRs-9 monoclonal antibody labelled with FITC.

The results above showed indication that TLR expression by epithelial cells in oral mucosa had remarkable implication on innate immune response and disease pathogenesis.

\section{DISCUSSION}

TLRs are type I transmembrane protein involved in innate immunity. Their structures are characterized by extracellular leucine-rich repeats and an intracellular motif with a high degree of homology to the intra-cellular domain of the IL-1 receptor. ${ }^{3,4}$

TLRs can specifically recognize pathogen-associated molecular pattern (PAMPs), transducer signals into cells and initiate complex signal cascades leading to activation of the transcriptional factors such as nuclear factor kappa $\mathrm{B}(\mathrm{NF}-\mathrm{k} \beta)$ and interferon regulatory factor. Subsequently, the inflammatory mediators such as IL-1a/b, IL-6, IL-8 and TNF- $\alpha$ are synthesized and released to activate neutrophils and lymphocytes. This result in the initiation of innate and adaptive immune response.

Deficiency of TLRs may result in corresponding pathogen recognition failure and susceptibility to certain pathological microbes. Hyper-expression of TLRs in infected tissues may promote excessive inflammation. ${ }^{18}$

In the family of 11 TLRs, the function of TLRs-10 remains unknown and the expression of TLRs 11 is limited to urethral epithelial cells. To further investigate the expression of TLRs in oral mucosa epithelium, the expression of TLR1-9 were studied. Molecule of pathogen can express some PAMPs, it is recognized of different TLRs, that heredity redundancy on immune system for blocking of microbial infection.

PAMPs come from positive gram organism (peptydoglycan and lipoprotein), it is recognized TLRs-2. The other member subfamily of TLRs- 2 is TLRs- 1 and TLRs-6. TLRs- 2 combined with TLRs- 1 and TLRs-6, that have a signal response to many kind of microbial pathogen as well as mycobacterium. TLRs-2 signal is needed for fragmentation of M. tuberculosis in macrophage. TLRs-2 in mouse has important role because the individual more sensitive to $\mathrm{M}$ tuberculosis infection if they have decrease of TLRs-2. On the otherhand, TLRs-2 also can bind components of herpes virus, which binds in turn to CD14 on the cell surface. ${ }^{19}$ RAS in Dental Hospital of Faculty of Dentistry at Airlangga University more $43,7 \%$ is found in mayor RAS. This phenomenon is very important in oral medicine because the signaling through TLRs occurs through a well described pathway in which receptor binding generates a signal through an adaptor molecule, MyD88, that leads to intracellular associated with IL-1 receptorassociated kinase.

TLRs-4 can mediated LPS as signaling from bacteria of gram negative that activated macrophage through TLRs- 2 . Base on the type of agent of microbial and their PAMPs that the TLRs can recognize direct to plasma membrane as well as TLRs-4 or direct in the phagosome like TLRs-2. TLRs response can appear in different cell expression. Epithelial gut cells using LPS that can associated with TLRs-4 in golgy complex in plasma membrane. This statement like with TLRs which expressed on mouth epithelial cells. However, on the basis of studies with TLRs-4 is similar 
but not identical to the signaling pathway activated by other TLRs. Also the activation of cytokine production by TLRs plays an important role in recruiting other components of innate host defense against molecule pathogen as well. ${ }^{19}$

It showed here that TLRs- 2 and TLRs-4 cells were present in ulcer tissues from both healthy control and subject with major and minor RAS. The presentation of TLRs-2 expression in major and minor RAS patients could be related with the presence of bacterial infection/LPS. Immunohistochemically, TLRs- 2 is an inflammation signaling in oral mucosa induced by gram-positive bacteria. Bacterial molecules that can induce TLRs-2 expression are outer membrane and polysaccharides. These molecules apparently had important role in RAS patients visiting dental outpatient clinic. Therefore, in oral cavity, the presence of bacterial infection should be noticed although the bacteria are not the predominant cause of RAS., $2,6,8$

There are some alternative of TLRs- 2 and TLRs- 4 that cause inflammatory process in correlation with RAS. The first alternative is through hyper secretion of cytokine, also can active an pro inflammation molecule as well as TNF- $\alpha$, IL-1 $\beta$, IL-6 and IL-12. The other molecules are activated too as accessories cells are neutrophil, dendritic cells using signaling molecule RANTES, MIP- $1 \alpha$ and MIP- $1 \beta$. The second alternative is inflammatory process began by special pathway, that called MyD88 dependent pathway using activated TIRAM and then induce IRAK-4 and TRAP6 and activated MAPKKK, these process as became signaling innate immunity through MAP3 and protein (p38), finally inducing AF-1 to make inflammation. ${ }^{8,19,20}$

Especially TLR2 has two possibilities of signaling, through RAC1 and MYD88. The signaling is through MYD88 so it can be activated FADD and then caspase and cause fragmented of DNA known as apoptosis. The signaling that through RAC1 pathway were activated through $\mathrm{P} 13 \mathrm{~K}$ and AKT to induce signal NF- $\kappa \beta$ (nuclear factor $\kappa \beta$ ) caused inflammatory. This pathway is predominant to cause RAS like TLRs-9 too used always MYD88 dependent pathway.

RAS identification using immunohistochemically examination revealed that TLRs- 2 was expressed at the surface of epithelial cells and macrophage, in addition to intra cytoplasm of epithelial cells. This was likely related to NF- $\kappa \beta$ activated through IFN-gamma, which finally lead to the occurrence of hypercytochemistry and ended with severe inflammation, resulting in RAS..$^{13-15,20}$

The properties of the TLRs- $3 \alpha$ is different with TLRs-2, TLRs- 4 with activity TLRs- 9 because TLRs- 3 can protect viral infection. The mechanism is began activation of TRIP and then activated TBK1 to give signal to IRF3 which finally secretie IFN- $\alpha, \beta$.

Interaction between LPS and receptor TLRs-4 induced complex signal that begin by activating of factors transcription such as $\mathrm{NF}-\kappa \beta$, and then stimulated and activated inflammation gene as TNF- $\alpha$, IL-6 and IL-8. TLR2 and TLR4 are known as mediate inflammatory responses to bacterial components. ${ }^{21}$ TLRs- 2 is responsible for the recognition of LTA, whereas TLRs-4 recognizes LPS in immune competent cells such as macrophages, the response to LPS is mediated by interaction with TLRs-4 in conjunction with TLRs-4 accessory protein MD-2 and CD 14, and transducers intracellular signals followed by the activation of TLRs-associated adapter protein, myeloid differentiation factor 88 (MyD88), leading to the activation of NF- $\kappa \beta .^{22}$

The samples which expressed TLRs-3 were mostly major RAS was $43.58 \%$ from 38 RAU patients, while those with minor RAS positive TLRs-3 was $17.94 \%$. Immunohistochemically examination using chromogene revealed that the samples showed significant reaction against TLRs-3, as it was clearly expressed at the surface of epithelial cells and macrophage, as it was also expressed within cytoplasm and intra cellular (intra nuclear). This indicated that the predominant inductor was a type of viral infection, with the result that cellular activities at molecular level, such as interleukin/the cytokines increased as well. TLRs-3 uses multiple mechanism to enhance and sustain the antiviral response more strongly than TLRs-4.

RAS patients with positive TLRs-4 was $48.71 \%$, found in major RAS patients, while in minor RAS TLRs4 was expressed $38.46 \%$. TLRs 4 could be detected on the surface of epithelial cells and cytoplasm from oral mucosal epithelium in either major or minor RAS patients. In major RAS patients, the TLRs-4 expression was more predominant in the surface of epithelial cell and macrophage. This seemed since the oral mucosal epithelium of patients with ulceration of oral mucosa can respond the presence of bacterial endotoxin by activating TLRs-2, TLRs-3, and TLRs-4.

TNF-alpha is an inflammatory cytokine produced through TLRs activation in its response against bacteria. ${ }^{23}$ TLRs with transmembrane protein and cell surface receptor and intra cytoplasms signal area may likely play more important role in this intracellular signaling. TLRs-4 blocking by neutralizing antibody anti-TLRs-4 apparently inhibited TNF-alpha after LPS administration. ${ }^{23}$ A previous study using immunohistochemistry also showed that TLRs- 2 and TLRs- 4 cells numbers increase in the inflamed mucosal ulcers. Although level of transcript were not quantitated.

\section{Expression of Toll-like receptors}

It has been proved immunohistochemically that TLRs can be detected on the surface of oral mucosal epithelium and cytoplasm of RAS patients. TLRs localization has been widely related with immune and inflammation cells. Epithelial cells in oral mucosa may relate with many potential pathogens, and TLRs expression will be relevant with immunity of oral mucosa. Epithelium is the primary target of infectious agents. Therefore, these epithelial cells play an important role in inflammation for production of various cytokines and pro inflammatory cytokines.

Preservation of IL-10, while IL- $1 \beta$ and TNF- $\alpha$ are down regulated, could play a protective role against inflammatory 
tissue destruction. On otherhand IL-1 $\beta$, TNF-alfa and IL-6 apart from being inflammatory mediators, also can facilitate soft and hard tissue destruction and favor the pathogenesis of RAS. ${ }^{14}$

Convergence of intracellular signaling pathways by TLRs and inflammatory cytokines (IL- $1 \beta$ and TNF- $\alpha$ ) along with reprogramming of signal transduction and gene transcription, may explain to certain extent the cytokine profile during endotoxin tolerance. The regulation of TLRs expression on macrophages in vitro is consistent with the inflammatory cytokine response. We showed that IL-1 $\beta$, TNF- $\alpha$, IL-6, IL- 8 and IL-10 levels increase on initial LPS stimulus but were not equally susceptible to a decrease on LPS challenge. Most notably IL-10 and IL-8 were refractory to endotoxin tolerance. TLRs mediate tolerance obtunds the inflammatory cytokine response. TLRs activation culminates in transcription of inflammatory cytokine which finally cause RAS. 5,14,20,25

In addition it was found that expressed TLRs were not only from one type, but also from the others. The predominant TLRs expressed in RAS patients were TLR-2, -3 and -4 . In connection with the result of previous studies in its clinical implications, the causing agents or inducers were not only one type, but an accumulation of molecules in oral mucosa that played mutual role as predominant inducer. Therefore, TLR was expressed not only on the surface of cell or macrophages, but also found in intra cytoplasms, surface membrane cells and even intra nucleus. ${ }^{20,24,25}$

The conclusions that TLRs expressed on the surface of oral mucosal epithelial cells and macrophages in RAS patients, either major or minor. TLRs was not specifically expressed in non-RAS. TLRs-2, TLRs-3 and TLRs-4 has been expressed on the surface of cells membrane and macrophages in minor and major RAS. TLRs was more predominantly expressed in major RAS compared to minor RAS. Functional TLR expression by oral mucosa epithelial cells had higher implications towards natural immune response and disease pathogenesis. It is suggested to undertake molecular characterization to determine specific TLR against specific disease agents, so that it might be easy to identify the causing agent, with the result that RAS management can be established comprehensively.

\section{ACKNOWLEDGEMENT}

The author would like to thank to Badan Litbangkes Departemen Kesehatan RI and Lembaga Biologi Molekuler Eijkmann which had given the sponsorship to do this research. We also thanks to Prof. Dr. Yoes Prijatna Dachlan, dr., M.Sc and Prof. Dr. Fedik A Rantam, drh. for valuable comments and discussions and Mrs. Helen for skillful technical help.

\section{REFERENCES}

1. Diamond GD, Legarda A, Ryan IK. The innate immune response of the respiratory epithelium. Immunol Rev 2004; 173:27-38.

2. Sugawara S, Uehara A, Tamai R, Takada H. Innate immune responses in oral mucosa. J Endotoxin Res 2002; 8(6):465-8.

3. Takeda K, Akira S. TLR signaling pathway. Semin Immunol 2004; 16:3-9.

4. Akira S, Hemmi H. Recognation of Pathogen-associated molecular pattern by TLR family. Immunol Lett 2003; 85:85-89.

5. Lewkowicz N, Lewkowicz P, Kumatowska A. Innate immune system is implicated in recurrent aphthous ulcer pathogenesis. J.Oral Pathol Med 2003; 32:475-81.

6. Delneste Y, Beauvillain C, Jeannin P. Innate immunity: structure and function of TLRs. Med Sci 2007; 23(1):67-73.

7. Beutler B, Rehli M. Evolution of the TIR, tolls and TLRs functional inferences from computational biology. Curr Top Microbial Immunol 2002; 270:1-21.

8. Medzhitov R. Toll-like receptor and innate immunity. Nat Rev Immunol 2002; 1:135-45.

9. Lorenz E. TLR2 and TLR-4 expression during bacterial infection. Curr Pharm 2006; 12(32):4185-93.

10. Mc. Nally. Recurrent aphthous stomatitis and precieved stress: Preliminary study. Available at: http:/www.aphthous.stress study. tripod.com/resed.doc. Accessed March 18, 2003.

11. Scully C, Gorsky M, Lozada-Nur F. The Diagnosis and management of recurrent aphthous stomatitis a consensus approach. J Am Dent 2003; 134(2):200.

12. Porter S, Scully C. Aphthous ulcers (recurrent). Clin Evid 2004; (11):1766-73.

13. Natah SS, Hayrinen-Immonen R, Hietanen J, MalmstromM, Konttinen YT. Immunolocalization of TNF- $\alpha$ expressing cells in RAU. J Oral Pathol Med 2000; 29:19-25.

14. Sun A, Chang YF, Chia JS, Chiang CP. Serum IL-8 level is a more sensitive marker than serum IL-6 level in monitoring the disease activity of RAU. J Oral Pathol Med 2004; 33:133-9.

15. Hasan A, Shinnick T, Mizushima Y, Van Der Zee R, Lehner T. Defining T-cell epitope within HSP65 in RAS. Clin Exp Immunol 2002; 128:318-25.

16. Ernawati DS. Immune response $65 \mathrm{kDa}$ protein in recurrent aphthous ulceration (RAU). The International Journal of Oral Health 2004; 1:53. Abstract.

17. Ernawati DS. The molecular analysis on the expression of protein anomaly oral mucosa in RAS patients. Indonesian Journal of Dentistry 2006; 13(Special Edition KPPIKG XIV).

18. Borra RC, Andrade PM, Silva IDCG, Morgan A, et al. The Th1/Th2 immune-type response of the recurrent aphthous ulceration analyzed by cDNA microarray. J Oral Pathol Med 2004; 33:140-6.

19. Tosi MF. Innate immune responses to infection. J Allergy Clin Immunol 2005; 116(2):241-7.

20. Wu Xin-yi, Gao Jian-lu and Ren Mei-yu. Expression profiles and function of Toll-like receptors in human corneal epithelia. Chinese Medical Journal 2007; 120(10): 893-7.

21. Anderson KV. Toll signaling pathways in the innate immune response. Curr Opin Immunol 2003; 12:13-19.

22. Kaisho T, Akira S. Toll-like receptors as adjuvant receptors. Biochem Biophys Acta 2002; 1589:1-13.

23. Xu Z, Dzarski R, Wang Q, Swartz K, Sakamoto KM, Gupta D. Bacterial peptidoglycan-induced TNF-alpha transcription is mediated through the transcription factors Egr-1, ELK-1 and NF-kappa Beta. J Immunol 2001; 167:6975-82.

24. Stefan B, Gunther H. Toll-like receptors (TLRs) and innate immunity. Handbook of Experimental Pharmacology 183. Springer-Verlag Berlin Heidelberg; 2008. p. 1-14.

25. Cario E, Gerken G, Podolsby DK. Toll like receptors-2 control mucosal inflammation by regulating epithelial barrier function. Gastroenterology 2007; 132(4):1359-74. 